\title{
State of Charge Based Droop Control for Coordinated Power Exchange in Low Voltage DC Nanogrids
}

\author{
Cephas Samende, Sivapriya M. Bhagavathy, Malcolm McCulloch \\ Department of Engineering Science \\ University of Oxford, Oxford, UK \\ \{cephas.samende, sivapriya.mothilalbhagavathy, malcolm.mcculloch@eng.ox.ac.uk\}
}

\begin{abstract}
Decentralized battery and solar photovoltaic (PV) system organized in the form of an autonomous low voltage DC nanogrid is a potentially low cost and scalable solution for electrifying rural areas without access to the national grid. Each DC nanogrid can be installed on a single home and used to supply basic lighting, charge mobile phones and power a television set. To provide enough power to meet productive energy uses such as irrigation, the DC nanogrid can be connected to neighboring DC nanogrids to form a cluster and exchange power. However, to achieve a coordinated power exchange in the cluster, new control strategies are required. In this paper, we propose a decentralized droop control method which uses a state of charge of the battery to coordinate the power exchange. The power exchange is achieved by scheduling a terminal voltage set point at each DC nanogrid based on the state of charge of the battery. The performance of the proposed method at achieving the power exchange is analyzed through simulations in Matlab/Simulink. The method does not require inter-unit communication. Therefore, the method is reliable, robust and scalable. Also, the method maintains low amounts of power flow in distribution lines during power exchange to reduce distribution line power losses.
\end{abstract}

Keywords- Low Voltage DC Nanogrid, Cluster, Power Exchange, State of Charge, Droop Control, Power Losses, Rural Areas

\section{INTRODUCTION}

In this paper, we address the challenge of achieving a coordinated power exchange in a cluster of low voltage DC nanogrids (DCNGs) used for rural electrification. According to energy access outlook 2017, around 1.1 billion people in the world do not have access to electricity. Eighty percent of them live in rural areas of sub-Saharan Africa [1]. Lack of access to electricity deprives the rural communities of accessing the basic needs of life such as quality education and health delivery. As most rural areas are sparsely populated, electrification by extending the national grid is expensive.

Due to the declining costs of solar photovoltaic (PV) modules [2], off-grid decentralized power systems (DCNGs) with PV systems are an economical alternative to providing electricity access to the rural areas. Each DCNG consists of a battery for energy storage, power electronics converter(s) for an interface and a solar PV system for energy generation [3]. Each DCNG can be installed on a single home and used to supply basic lighting, charge mobile phones and power a television set. To enhance power supply reliability and to provide enough power to meet productive energy uses such as irrigation, each DCNG can be connected to neighbouring DCNGs to form a cluster and exchange power. The energy surplus or deficit in one DCNG get balanced by the neighbouring DCNG. This eliminates the need for other expensive options of enhancing supply reliability such as diesel gensets. However, due to the decentralized batteries and intermittent nature of the PV in the cluster, control, and coordination of the power exchange is a challenge.

In [4]-[6], a hierarchical control approach of the cluster for power exchange is proposed. Despite the effective power exchange coordination among the DCNGs, the approach uses a communication link for control. The use of a communication link presents a single point of failure and increases the cost of the power network. Therefore, a decentralized control strategy that does not depend on communication for operation is preferred.

State of charge (SoC) based droop control is a new decentralized control strategy aimed at controlling DCNGs with decentralized batteries. The existing SoC based droop control strategies can be grouped into two categories: SoC weighted droop gain and SoC weighted nominal DC bus voltage.

SoC weighted droop gain method is proposed in [7], [8] to achieve balanced SoC among distributed batteries supplying a common load. With this method, the droop gain is changed based on the SoC of the battery to set the voltage of a single battery unit. Despite good performance in achieving balanced SoC, the approach suffers from system instability at low SoC values during charging and high SoC values during discharging when the droop gains are small [9].

To overcome the system instability, SoC weighted nominal DC bus voltage method has been proposed in [9][11]. With this method, the nominal DC bus voltage is changed based on SoC. However, for a network with sparsely located batteries, this would require a communication link to measure the DC bus voltage. The use of communication increases the cost of the network and presents a single point of failure.

In this paper, we propose to schedule the terminal voltage of a single DCNG based on SoC of the battery in order to achieve a coordinated power exchange in the cluster. The droop gain and nominal DC bus voltage are kept constant in the proposed strategy. 


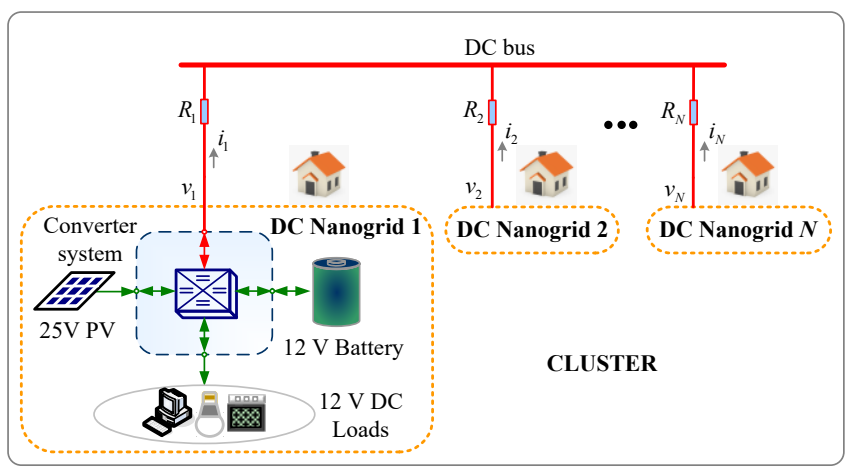

Fig. 1. Schematic diagram of a cluster with $N$ DCNGs (homes), each having a $25 \mathrm{~V} \mathrm{PV}$ panel, $12 \mathrm{~V}$ battery, $12 \mathrm{~V}$ DC local loads, four port DCDC converter system and an external resistive distribution line, $R_{k} . k=1$, $2, \cdots, N$.

The transient response analysis of the cluster when DCNGs start to exchange power also forms part of the contribution of this paper.

The rest of the paper is organized as follows: Section II describes the cluster considered in this paper. The proposed control strategy is detailed in Section III. Section IV analyses the dynamic transient response of the network to differences in SoC, battery size and line resistances. Simulation results are discussed in Section V and Section VI concludes the paper.

\section{STRUCTURE OF CLUSTER}

Fig. 1 shows the schematic diagram of the cluster considered in this paper. It consists of $N$ DCNGs, each with a rooftop mounted PV panel, battery, DC loads (e.g. TV, computer and LEDs) and a four port DC-DC converter system. The four port DC-DC converter topology is shown in Fig. 3 and consist of a high frequency transformer (HF-T) to provide galvanic isolation and voltage transformation among the DCNG components. The PV operates in maximum power point tracking (MPPT) mode to fully exploit the variable PV power. The DCNGs are connected to each other via distribution lines; $R_{l}, R_{2}, \cdots, R_{N}$. During operation and assuming a lossless converter, the laws of physics require that the power balance in (1) is satisfied at each DCNG.

$$
P_{k}^{b}(t)=P_{k}^{L}(t)-P_{k}^{p v}(t)+P_{k}^{g}(t)
$$

where $k$ is the DCNG number, $P_{k}^{b}(t)$ is the battery charge/discharge power, $P_{k}^{L}(t)$ is the local load, $P_{k}^{p v}(t)$ is the PV power output, and $P_{k}^{g}(t)$ is the power exchanged with other DCNGs. From (1), the amount of energy present in the battery at every time slot, $t$ is related to its $\mathrm{SoC}$ as [12]

$$
\operatorname{SoC}_{k}(t)=\operatorname{SoC}_{k}(0)-\frac{1}{W_{k}} \int_{t=0}^{T} P_{k}^{b}(t) d t
$$

where $W_{k}$ is the battery nominal energy capacity in $\mathrm{Wh}$, $\mathrm{SoC}_{k}(0)$ is the initial SoC, and $T$ is the period of energy accumulation in hours. Substituting (1) in (2) yields:

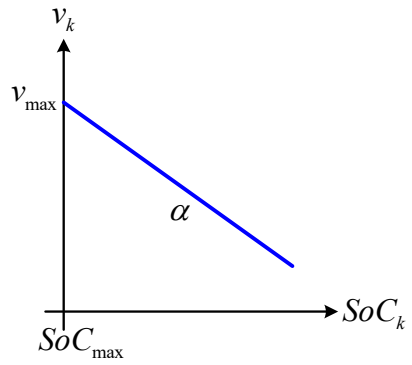

Fig. 2. Proposed voltage - state of charge voltage droop control

$$
S o C_{k}(t)=S o C_{k}(0)-\frac{1}{W_{k}} \int_{t=0}^{T}\left[P_{k}^{L}(t)-P_{k}^{p v}(t)+P_{k}^{g}(t)\right] d t
$$

where $V_{k}^{b}$ is the rated battery voltage. From (3), the SoC can be used as a signal to indicate the amount of energy resources available in each DCNG at every instant of time. The higher the SoC, the higher the amount of available energy in the DCNG. The converse is also true.

\section{PROPOSED CONTROL STRATEGY}

In this section, the proposed voltage - state of charge $(\mathrm{V} / \mathrm{SoC})$ droop control method is detailed. The method is shown in Fig. 2 and can be expressed as

$$
v_{k}=v_{\max }-\alpha\left(S o C_{\max }-S o C_{k}\right)
$$

where $v_{\max }$ is the maximum voltage of the cluster, $\alpha$ is the droop gain, $S o C_{k}$ is the estimated $\mathrm{SoC}$ and $S o C_{\max }$ is the maximum $\mathrm{SoC}$ when the battery is fully charged.

The method sets the terminal voltage, $v_{k}$ of DCNG $k$ based on $S o C_{k}$. A high SoC value would mean that the DCNG has high amounts of available energy and would have its terminal voltage set higher than others. Conversely, for a low SoC value, the DCNG is said to have low amounts of available energy and would have its terminal voltage set lower than others. Due to high $R / X$ ratio in DC networks [13], power will automatically flow from the DCNG with a high voltage setting to neighboring DCNGs with relatively low voltage settings. The power exchange stops when all the terminal voltages in the cluster converge to the same voltage value (i.e. when the SoCs in the cluster get balanced).

To ensure balanced SoCs in the cluster when there is no power exchange, $\alpha$ is kept constant in the droop equation (4). Any slight change in the SoC triggers power exchange. As the result, the amount of power exchanged at every instant of time is low and the distribution line losses are kept low.

For a typical maximum $\pm 10 \%$ voltage regulation, the droop gain can be selected to satisfy (5)

$$
\alpha \leq \frac{v_{\max }-v_{\min }}{S o C_{\max }-S o C_{\min }}
$$

where $v_{\min }$ and $S o C_{\min }$ is the minimum terminal voltage and minimum SoC respectively. The control structure of the proposed method is shown in Fig. 3. 


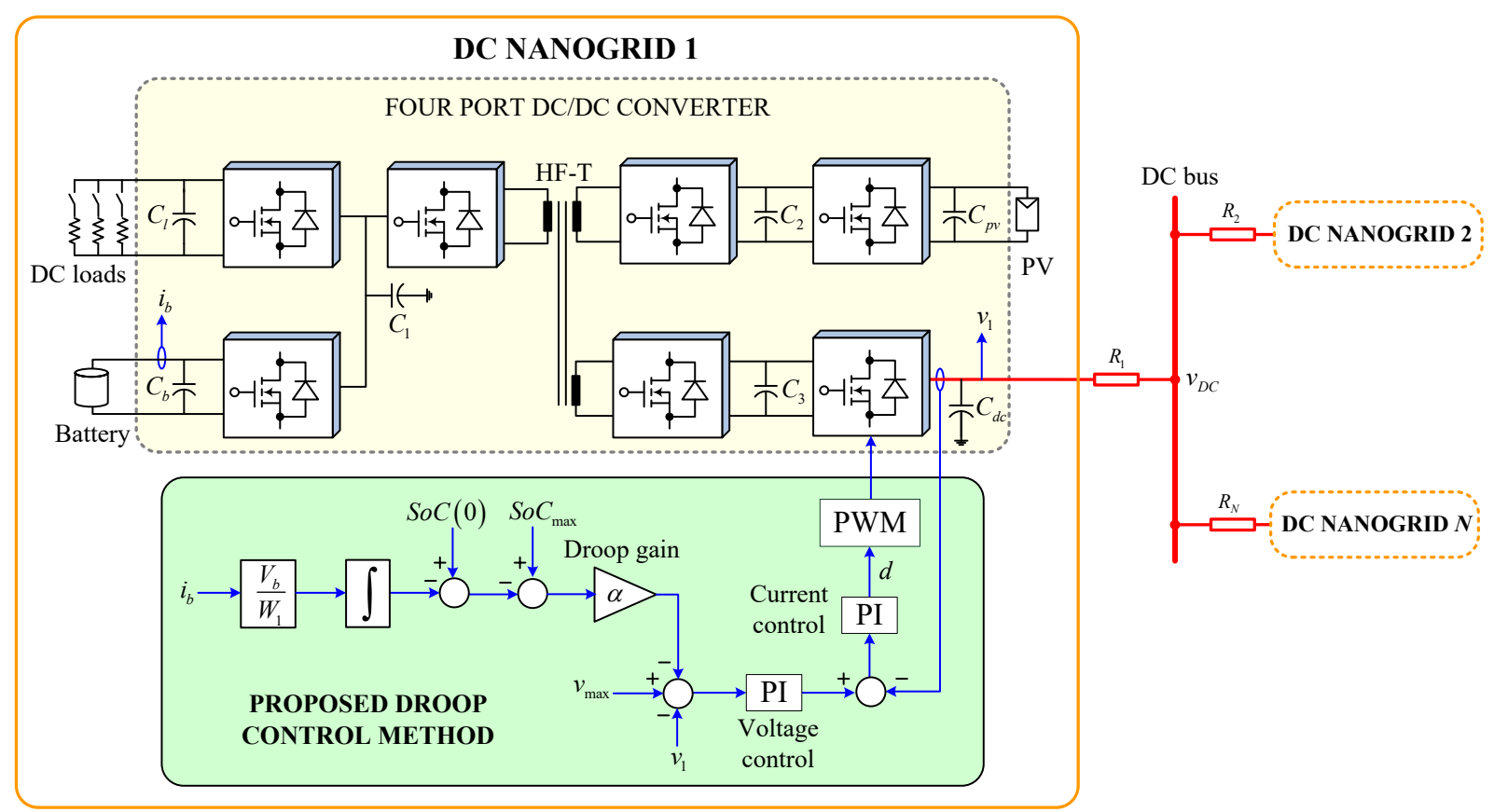

Fig. 3. Control structure of the proposed droop control method that sets the terminal voltage $v_{l}$ based on battery SoC for a single DCNG 1 in order to achieve a coordinated power exchange with the neighbouring DCNGs; 2, 3, .$\cdot N$.

\section{TRANSIENT RESPONSE ANALYSIS}

In this section, the transient response of the cluster when the DCNG is connected to neighboring DCNGs to exchange power is analyzed. Knowledge of the transient response can help us understand what would happen to the voltage and current in the distribution line if a new DCNG is added to the cluster.

Since in this work, power is exchanged each time there is a change in the SoC, effectively and without loss of generalization, each DCNG can be represented by a battery unit in parallel with a DC load, $R_{L}$ as shown in Fig. 4 (a). From Fig. 4(a), closing switch $s_{l}$ at time $t=0$ s represents the battery charge and discharge from and to the neighboring DCNGs respectively. Whereas closing switch $s_{2}$ represents the battery discharge to supply the load, $R_{L}$.

Omitting the converter losses, the battery charge/discharge current, $i_{b}$ and power at $t=0 \mathrm{~s}$ is related to the distribution line current, $i_{k}$ as

$$
\begin{aligned}
& i_{b}=n i_{k} \\
& P_{k}^{b}=i_{b} V_{b}=n i_{k} V_{b}
\end{aligned}
$$

where $n$ is the converter current transformation ratio and $V_{b}$ is the rated battery voltage. Substituting (7) in (2) and the resulting expression in (4) yields

$$
v_{k}=v_{\max }-\alpha\left(S o C_{\max }-S o C_{k}(0)\right)-\frac{n \alpha V_{b}}{W_{k}} \int_{t=0}^{T} i_{k}(t) d t
$$

Representing

$$
q_{k}(t)=-\int_{t=0}^{T} i_{k}(t) d t
$$

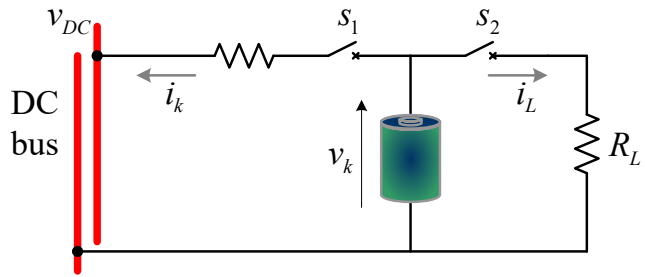

(a)

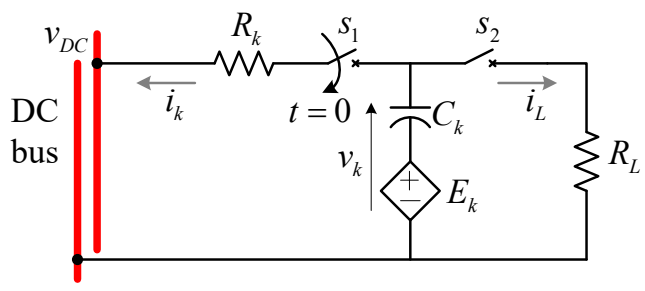

(b)

Fig. 4. Equivalent circuit of DC nanogrid during transient response (a) battery equivalent and (b) resistor capacitor (RC) equivalent

expression (8) can be expressed as a dependent voltage source (a function of initial SoC) in series with a capacitor voltage (10).

$$
v_{k}=E_{k}+\frac{q_{k}(t)}{C_{k}}
$$

where,

$$
\left\{\begin{array}{l}
E_{k}=v_{\text {max }}-\alpha\left(S o C_{\text {max }}-S o C_{k}(0)\right) \\
C_{k}=\frac{W_{k}}{n \alpha V_{b}}
\end{array}\right.
$$

It is clear from (10) and (11) that using the droop equation (4) to set the terminal voltages of the DCNGs makes each DCNG behave like a capacitor during the transient period (Fig. 4(b)). Therefore, Fig. 4(b) can be analyzed at $t=0 s$ (with $s_{2}$ open) to obtain the transient 
current response, $i_{k}$ during battery charge and discharge from and to the neighboring DCNGs respectively.

For a cluster (Fig. 1) with three (3) DCNGs, $i_{k}$ during battery discharge (which is symmetrical to battery charge) can be derived as an overdamped current response (12).

$$
i_{k}(t)=A_{k} e^{-\zeta \omega_{n} t}\left\{\begin{array}{l}
{\left[\omega^{*} \sinh \left(\omega^{*} t\right)+B_{k} \omega^{*} \cosh \left(\omega^{*} t\right)\right]} \\
-\zeta \omega_{n}\left[\cosh \left(\omega^{*} t\right)+B_{k} \sinh \left(\omega^{*} t\right)\right]
\end{array}\right\}
$$

where the terms are defined and given in Appendix I.

With (12), the effect of connecting a DCNG to other DCNGs at $t=0 s$ on current transient response either during power exchange or when it is newly added to the cluster can be studied. Similarly, the voltage transient response can be studied and is beyond the current scope.

\section{a. Influence of Line Resistance on Transient Current Response}

Fig. 5 shows the influence of the distribution line resistance on the transient current response of the DCNG. From the figure and intuition, high transient currents are obtained when a low resistive line is used as shown in Fig. 5(a). The magnitude and speed of the transient current response reduce for high resistive lines as shown in Fig. 5(b) - Fig. 5(d).

\section{b. Influence of Battery Size on Transient Current Response}

The influence of battery size on magnitude and speed of the transient current response is shown in Fig. 6. We can observe that the battery size only influences the speed of the response for the given DCNG parameters. The speed reduces for bigger batteries as shown in Fig. 6(b)-Fig. 6(d).

\section{c. Influence of Initial State of Charge on Transient Current Response}

Fig. 7 shows the effects of connecting DCNGs with different initial battery SoCs on the transient current response. As shown in Fig. 7(a) - Fig. 7(d), low current magnitudes and high response speeds are obtained when the difference between the initial SoCs is small.

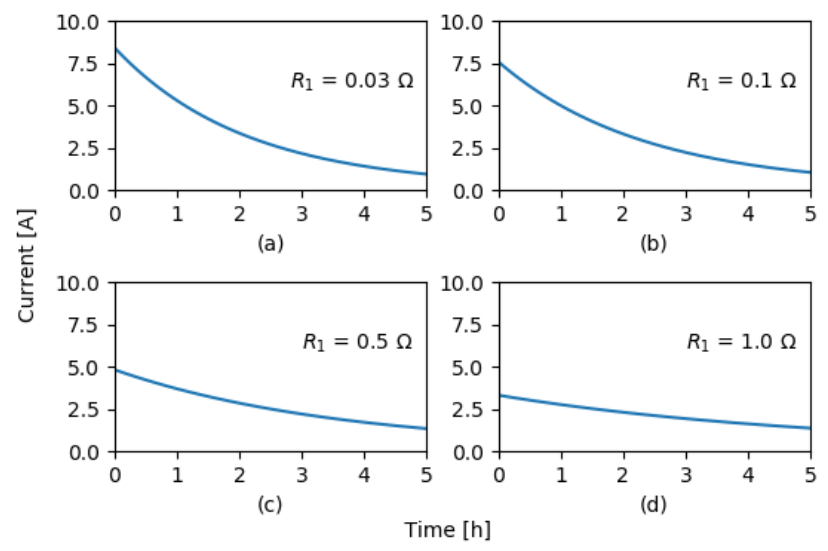

Fig. 5. Influence of line resistance on transient current response either during power exchange or when a new DCNG is added to the cluster.

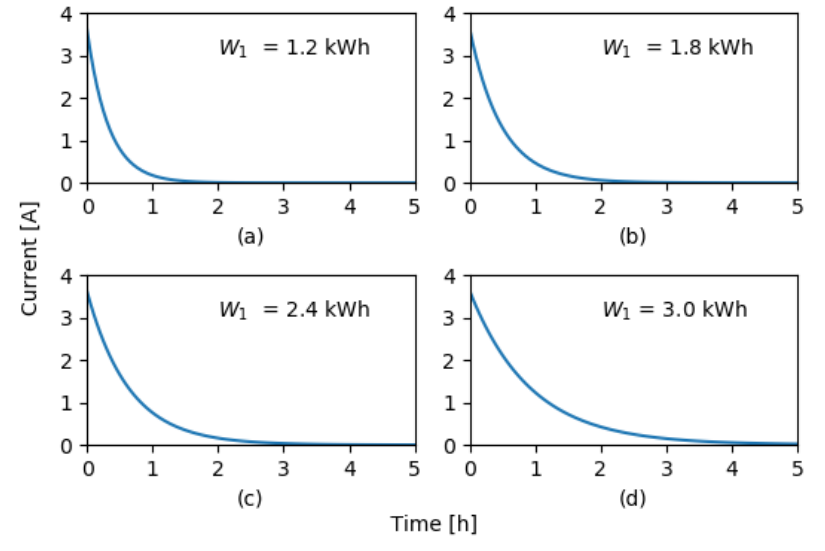

Fig. 6. Influence of battery size on transient current response either during power exchange or when a new DCNG is added to the cluster.

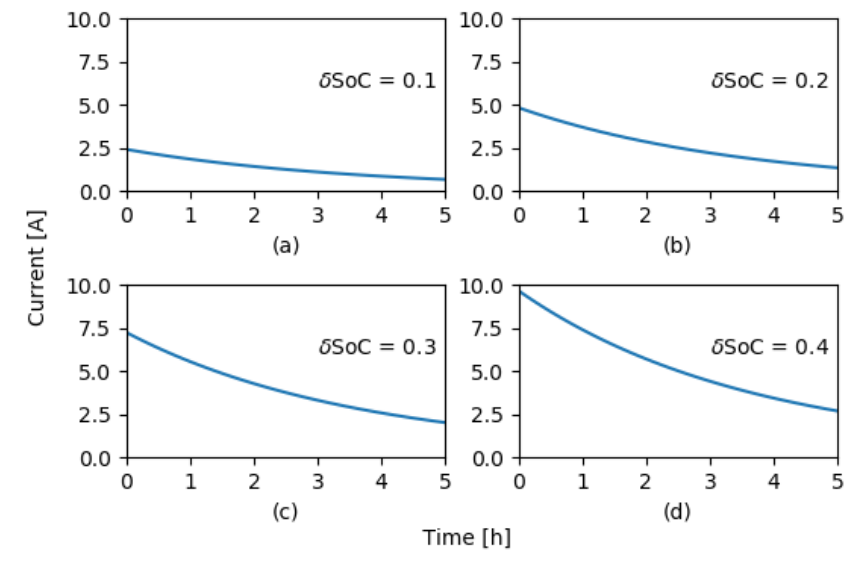

Fig. 7. Influence of SoC difference between DCNG batteries on transient current response either during power exchange or when a new DCNG is added to the cluster.

TABLE I

SIMULATION PARAMETERS

\begin{tabular}{|l|l|l|}
\hline PARAMETER DESCRIPTION & SYMBOL & VALUE \\
\hline \hline Battery capacity & $W_{1}, W_{2}, W_{3}$ & $1.2 \mathrm{Wh}$ \\
\hline Distribution line & $R_{1}, R_{2}, R_{3}$ & $0.9 \Omega$ \\
\hline Droop gain & $\alpha$ & $26.7 \mathrm{~V}$ \\
\hline Maximum voltage & $v_{\max }$ & $130 \mathrm{~V}$ \\
\hline
\end{tabular}

\section{Simulation RESUltS AND DISCUSSION}

Fig. 1 with three (3) DCNGs was used to verify the performance of the proposed droop control in Matlab/Simulink. The PV was modelled as a constant current source and the battery as a constant voltage source. The DC loads were modelled as resistors in parallel and an average model of the four port DC - DC converter was used for simulation. The converter topology and simulation parameters are detailed in [14]. Other key simulation parameters are shown in Table I. To speed up the simulation, similar and small battery sizes were considered. To verify the performance of the proposed control method for various operating conditions of the cluster, the loads and PV power were sequentially increased in each DCNG. As the result, the following operating scenarios were obtained in each DCNG and were analysed: 


\section{(a) Load Consumption Equal PV Generation}

This scenario is shown in Fig. 8 from $t=0 \mathrm{~s}$ to $t=10 \mathrm{~s}$. Due to the different DCNG initial SoCs as shown in Fig. 8(e), different terminal voltage setpoints are established as shown in Fig. 8 (d). DCNG 3 with high initial SoC has the highest initial terminal voltage setting and DCNG 2 with the lowest initial SoC has the lowest voltage setting. Consequently, high transient power flow from DCNG 3 with the highest terminal voltage setting to charge the battery in DCNG 2 with the lowest terminal voltage setting. As a result, the SoC of DCNG 3 decreases whereas that of DCNG 2 increases as time approaches $t=10 \mathrm{~s}$. At $t=10 \mathrm{~s}$, all the SoC and terminal voltages converge to 0.6 and $123 \mathrm{~V}$ respectively and power exchange ceases to occur.

\section{(b) Load Consumption Exceed PV Generation}

This case is shown in Fig. 8 from $t=10 \mathrm{~s}$ to $t=40 \mathrm{~s}$. It represents a typical operation during night time where energy stored in the batteries is exchanged between the DCNGs. At $t=10 \mathrm{~s}$, the load in DCNG 1 was increased from $76.4 \mathrm{~W}$ to $100.4 \mathrm{~W}$ whereas the loads in DCNG 2 and DCNG 3 were kept constant at $76.4 \mathrm{~W}$ and $131 \mathrm{~W}$ DCNGs. As the result, the local battery in DCNG 1 was used to supply the deficit and its $\mathrm{SoC}$ and the terminal voltage decreased as shown Fig. 8(e) and Fig. 8(d). Due to the difference in voltage potential, power automatically flows from DCNG 2 and DCNG 3 (with the same higher terminal setting) to DCNG 1 with a low voltage terminal setting. The exchanged power was used to meet the load demand only as shown in Fig. 8(a). Due to the same DCNG initial SoCs at $t$ $=10 \mathrm{~s}$, the power flow in the distribution lines was kept low and the distribution line losses can be minimised. Similarly, when the loads in DCNG 2 were increased at $t=20 \mathrm{~s}$ in Fig. $8(a)$, the local battery in DCNG 3 was used to meet both the deficit in DCNG 1 and DCNG 2. When the loads were increased in DCNG 3 at $t=30 \mathrm{~s}$ as shown in Fig. 8(c), the battery in each DCNG was prioritised to supply the local loads with little participation in power sharing.

\section{(c) PV Generation Exceed Load Consumption}

This situation is shown in Fig. 8 from $t=40 \mathrm{~s}$ to $t=68 \mathrm{~s}$ and represents an operation during day time when PV generation exceeds the load demand and the excess is used to charge the batteries. At $t=40 \mathrm{~s}$, PV generation was increased from $76.4 \mathrm{~W}$ to $166 \mathrm{~W}$ in DCNG 1 as shown in Fig. 8(a). From the figure, the surplus is used to charge the local battery and supply the loads in DCNG 2 and DCNG 3 as shown in Fig. 8(b) and Fig. 8 (c). The supplied power is just enough to meet the load demand in DCNG 2 and DCNG 3 and as the result, the batteries are idle between $t=$ $40 \mathrm{~s}$ and $t=50 \mathrm{~s}$ as shown in Fig. 8(b) - Fig. 8(e). At $t=50 \mathrm{~s}$ and $t=60 \mathrm{~s}$, the PV generation in DCNG 2 and DCNG 3 was increased as shown in Fig. 8(b) and Fig. 8(c) respectively. The surplus was used to charge the batteries as represented by the rise in the DCNG SoCs and terminal voltages in Fig. 8(d) and Fig. 8(e) respectively.

From the above results, we have shown that the proposed control strategy can be used to manage the power exchange in a cluster of DCNGs without the use of any logical communication. In addition, it has been shown that by allowing simultaneous power exchange from the DCNGs, low amount of power flow in the distribution lines can be

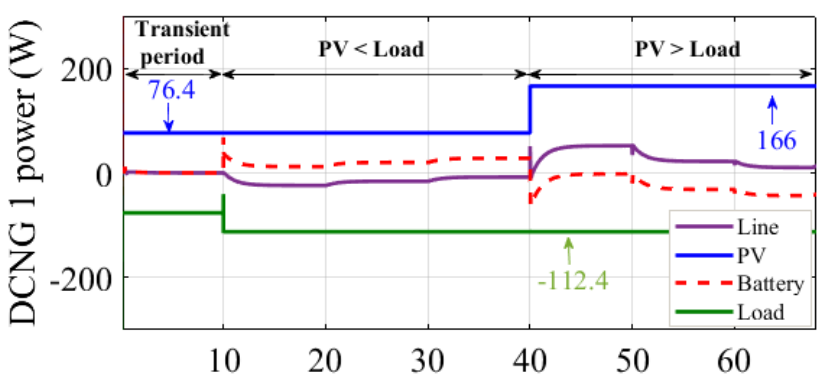

(a)

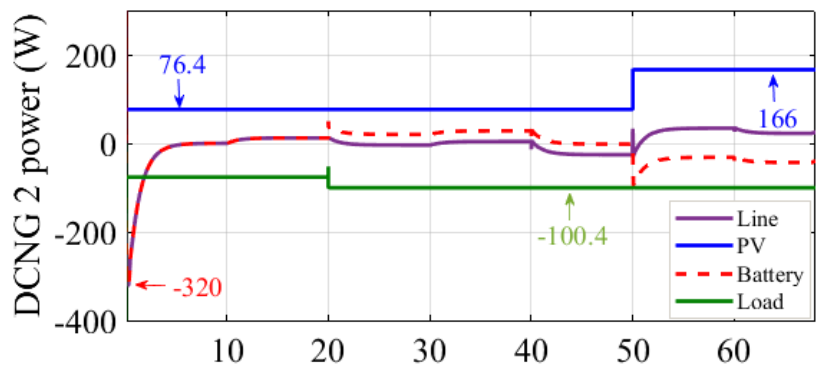

(b)

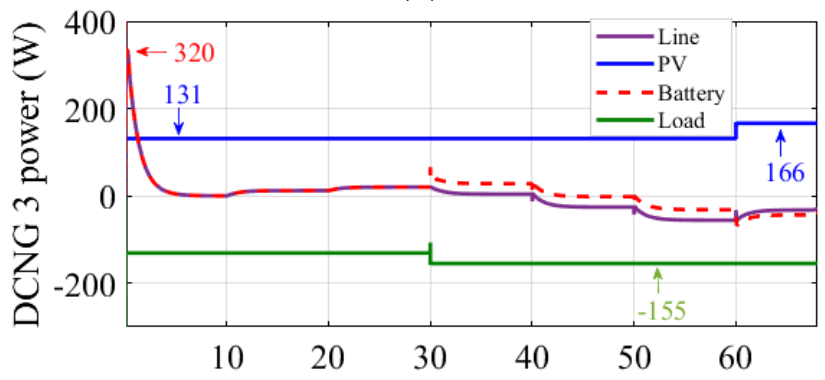

(c)

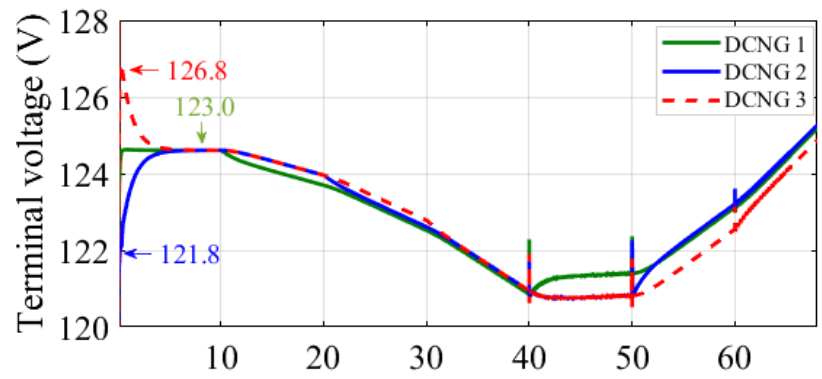

(d)

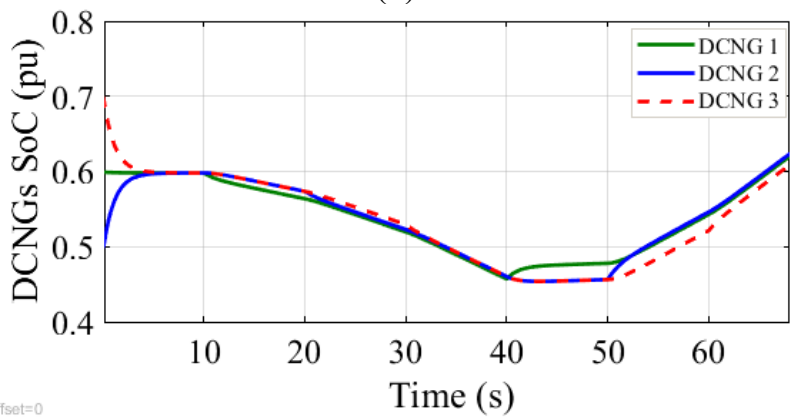

(e)

Fig. 8. Performance of the proposed control method at achieving coordinated power exchange and reduced amounts of power flow in the lines among three DCNGs: (a) DCNG 2, (b) DCNG 2, and (c) DCNG 3. The terminal voltage and $\mathrm{SoC}$ response are given in (d) and (e) respectively.

obtained. As a result, distribution power losses are reduced. However, the proposed method exhibits excessive transient power flow during starting for distribution lines with low resistance and when the battery SoCs are different. 
Excessive voltage and current during starting can damage cable insulations and power electronic equipment. Therefore, in our future work we plan to modify the proposed droop equation to ensure reduced current magnitudes and increased response speed during starting.

\section{CONCLUSION}

A decentralized $\mathrm{V} / \mathrm{SoC}$ droop control method was proposed in this paper to coordinate power exchange in a cluster of low voltage DC nanogrids. Power exchange was achieved by scheduling the terminal voltages of the DC nanogrids according to the state of charge of their local batteries. As the result, power automatically flows from DC nanogrids with high terminal voltages to those with low terminal voltages. The method maintains low amounts of power flow in the distribution lines during power exchange. Therefore, distribution line losses are reduced despite the frequent power exchange. The performance of the method for various operating conditions of the cluster was verified by simulations in Matlab/Simulink. The dynamic performance of the method when DC nanogrids with different battery size, start of charge and distribution line resistance are connected to each other was also analyzed. It was found out that the proposed method exhibits excessive transient power flow at the start of power exchange for distribution lines with low resistance and when the state of charge of the batteries were different. In our future work we intend to modify the proposed droop equation to ensure reduced current magnitudes and increased response speed during starting.

\section{APPENDIX I}

According to Kirchhoff's voltage law, the currents in each branch in Fig. 1 can be expressed as

$$
i_{k}(t)=\frac{v_{k}(t)-v_{D C}(t)}{R_{k}}
$$

where $v_{k}(t)$ is given by $(10)$ and

$$
v_{D C}(t)=\frac{\sum_{k=1}^{N} \frac{v_{k}(t)}{R_{k}}}{\sum_{k=1}^{N} \frac{1}{R_{k}}}
$$

The time domain differential equation representing (A.1) can be generated as

$$
-\frac{d q_{k}(t)}{d t}=\frac{v_{k}(t)-v_{D C}(t)}{R_{k}}
$$

Applying the Laplace Transform to (A.3) with rest $\left(q_{l}(0)=\right.$ $q_{2}(0)=q_{3}(0)=0$ ) initial conditions and solving the resulting equation for $N=3$ and $n=1$ yields (A.4)

$$
i_{k}(t)=A_{k} e^{-\zeta \omega_{n} t}\left\{\begin{array}{l}
{\left[\omega^{*} \sinh \left(\omega^{*} t\right)+B_{k} \omega^{*} \cosh \left(\omega^{*} t\right)\right]} \\
-\zeta \omega_{n}\left[\cosh \left(\omega^{*} t\right)+B_{k} \sinh \left(\omega^{*} t\right)\right]
\end{array}\right\}
$$

where,

$$
R_{e q}=R_{1} R_{2}+R_{2} R_{3}+R_{1} R_{3}
$$

$$
\begin{aligned}
& \zeta= \frac{C_{1} C_{2}\left(R_{1}+R_{2}\right)+C_{1} C_{3}\left(R_{1}+R_{3}\right)+C_{2} C_{3}\left(R_{2}+R_{3}\right)}{\sqrt{4 R_{e q}\left(C_{1} C_{2}^{2} C_{3}+C_{1}^{2} C_{2} C_{3}+C_{1} C_{2} C_{3}^{2}\right)}} \\
& \omega_{n}= \frac{\sqrt{R_{e q}\left(C_{1} C_{2}^{2} C_{3}+C_{1}^{2} C_{2} C_{3}+C_{1} C_{2} C_{3}^{2}\right)}}{C_{1} C_{2} C_{3} R_{e q}} \\
& \omega^{*}= \omega_{n} \sqrt{\left(\zeta^{2}-1\right)} \\
& A_{1}= \frac{C_{1}\left[C_{2}\left(E_{1}-E_{2}\right)+C_{3}\left(E_{1}-E_{3}\right)\right]}{C_{1}+C_{2}+C_{3}} \\
& C_{1} C_{2}^{2}\left(E_{2}-E_{1}\right)\left(R_{1}+R_{2}\right)+C_{1} C_{3}^{2}\left(E_{3}-E_{1}\right)\left(R_{1}+R_{3}\right) \\
&+C_{2} C_{3}^{2}\left[R_{2}\left(E_{1}-E_{3}\right)+R_{3}\left(E_{1}+E_{3}-2 E_{2}\right)\right] \\
&+C_{2}^{2} C_{3}\left[R_{3}\left(E_{1}-E_{2}\right)+R_{2}\left(E_{1}+E_{2}-2 E_{3}\right)\right] \\
&+C_{1} C_{2} C_{3}\left[R_{1}\left(E_{2}+E_{3}-2 E_{1}\right)+R_{2}\left(E_{1}-E_{3}\right)+R_{3}\left(E_{1}-E_{2}\right)\right] \\
& B_{1} C_{1} C_{2} C_{3} R_{e q} \omega^{*}\left[C_{2}\left(E_{1}-E_{2}\right)+C_{3}\left(E_{1}-E_{3}\right)\right]
\end{aligned}
$$

\section{REFERENCES}

[1] IEA, 'WEO-2017 Special Report: Energy Access Outlook', 2017. [2] A. Jäger-Waldau, 'European Commission PV Status Report 2017 ', 2017.

[3] D. Burmester, R. Rayudu, W. Seah, and D. Akinyele, 'A review of nanogrid topologies and technologies', Renew. Sustain. Energy Rev., vol. 67, pp. 760-775, 2017.

[4] J. M. Guerrero, A Self-Sustained and Flexible Control Strategy for Islanded DC Nanogrids without Communication Links, no. January. 2019.

[5] Q. Shafiee, T. Dragi'cevi', J. C. Vasquez, and J. M. Guerrero, 'Hierarchical Control for Multiple DC-Microgrids Clusters', IEEE Trans. Energy Convers., vol. 29, no. 4, pp. 922-933, 2014.

[6] M. Shahbazi, B. Kazemtabrizi, and C. Dent, 'Coordinated Control of DC Voltage Magnitudes and State of Charges in a Cluster of DC Microgrids', 2016 IEEE PES Innov. Smart Grid Technol. Conf. Eur., pp. 1-5, 2016.

[7] X. Lu, K. Sun, J. M. Guerrero, and J. C. Vasquez, 'DoubleQuadrant State-of-Charge-Based Droop Control Method for Distributed Energy Storage Systems in Autonomous DC Microgrids.', IEEE Trans. Smart Grid, vol. 6, no. 1, pp. 147-157, 2015.

[8] M. Nasir et al., 'A Decentralized Control Architecture applied to DC Nanogrid Clusters for Rural Electrification in Developing Regions', IEEE Trans. Power Electron.,pp. 1-1, 2018

[9] C. Li, T. Dragicevic, M. G. Plaza, F. Andrade, J. C. Vasquez, and J. M. Guerrero, 'Multiagent based distributed control for state-ofcharge balance of distributed energy storage in DC microgrids', in IECON Proceedings (Industrial Electronics Conference), 2014, pp. 2180-2184.

[10] D. Wu, F. Tang, T. Dragicevic, J. M. Guerrero, and J. C. Vasquez, 'Coordinated Control Based on Bus-Signaling and Virtual Inertia for Islanded DC Microgrids', IEEE Trans. Smart Grid, vol. 6, no. 6, pp. 2627-2638, 2015.

[11] R. Hu and W. W. Weaver, 'Dc microgrid droop control based on battery state of charge balancing', in 2016 IEEE Power and Energy Conference at Illinois, PECI 2016, 2016, pp. 1-6.

[12] T. Kim, S. Member, and W. Qiao, 'A Hybrid Battery Model Capable of Capturing Dynamic Circuit Characteristics and Nonlinear Capacity Effects', IEEE Trans. ENERGY Convers., vol. 26, no. 4, pp. 1172-1180, 2011

[13] T. L. Vandoorn, B. Renders, L. Degroote, B. Meersman, and L. Vandevelde, 'Active load control in islanded microgrids based on the grid voltage', IEEE Trans. Smart Grid, vol. 2, no. 1, pp. 127139, 2011.

[14] C. Samende, N. Mugwisi, D. J. Rogers, E. Chatzinikolaou, F. Gao, and M. Mcculloch, 'Power Loss Analysis of a Multiport DC - DC Converter for DC Grid Applications', in IECON 2018 44nd Annual Conference of the IEEE Industrial Electronics Society, Control and Instrumentation, 2018, pp. 1412-1417. 